\title{
Influence of C-Axis Inter Unit Cell Resonant Tunneling on the Spectral Function in Bilayer Cuprates
}

\author{
Ajay $^{{ }^{*}}$, B. S. Tewari $^{2}$, Govind ${ }^{3}$ \\ ${ }^{1}$ Physics Section, Department of Paper Technology, Indian Institute of Technology Roorkee, Saharanpur Campus, \\ Saharanpur, India \\ ${ }^{2}$ Department of Physics, University of Petroleum and Energy Studies, Dehradun, India \\ ${ }^{3}$ National Physical Laboratory, Dr. K. S. Krishnan Marg, New Delhi, INDIA \\ E-mail:ajphyfpt@iitr.ernet.in \\ Received January 21, 2011; revised March 16, 2011; accepted March 29, 2011
}

\begin{abstract}
The role of intra unit cell coupling along with inter unit cell resonant tunneling between the copper-oxygen planes on the electronic spectral function in normal state of bilayer high $T_{c}$ cuprates like $\mathrm{Bi}_{2} \mathrm{Sr}_{2} \mathrm{CaCu}_{2} \mathrm{O}_{8+\mathrm{x}}$ is investigated. The Hubbard model including terms representing hopping between the planes within the unit cell, and resonant tunneling between the planes in two adjoining cells is used along with the Green's function equation of motion approach to obtain an expression of spectral function. The spectral function at $(\pi, 0)$ point of the Brillouin zone is numerically calculated. It is found that the intra unit cell coupling lead to splitting of spectral peak especially close to $(\pi, 0)$ point, while the inter unit cell resonant tunneling lead to a broadening in the spectral function and suppression of bilayer splitting in the normal state. In the presence of finite electron correlations the inter unit cell tunneling induce strong broadening in the spectral features. The electron correlations and inter unit cell tunneling is important in determining the shape of the spectral function in doped bilayer cuprates. These results are viewed in terms of the existing ARPES measurements on bilayer cuprates.
\end{abstract}

Keywords: Multilayer Superconductors, Electronic Structure, Strongly Correlated Systems

\section{Introduction}

The angle resolved photoemission spectroscopic (ARPES) measurements [1,2] have emerged as dependable and useful tools for understanding the electronic structure of layered cuprates. In comparison to the single layer per unit cell material such as $\mathrm{La}_{2-\mathrm{x}} \mathrm{Sr}_{\mathrm{x}} \mathrm{CuO}_{4}$, the ARPES spectra of bilayer cuprates such as $\mathrm{Bi}_{2} \mathrm{Sr}_{2} \mathrm{CaCu}_{2} \mathrm{O}_{8+\mathrm{x}}(\mathrm{Bi}-2212)$ showed a splitting of spectral function along the antinodal point of the Brillouin zone. A number of experiments are performed to study the band splitting in Bi-2212 which has two $\mathrm{Cu}-\mathrm{O}$ planes within its unit cell [3-6]. The splitting is found to be large in normal state, with the maximum splitting $(\sim 110 \mathrm{meV})$ at $(\pi, 0)$ point of the Brillouin zone. It is also pointed out that the normal state of cuprates shows broadening in the spectral function while sharp and prominent spectral features are observed in the superconducting state. The splitting in the electronic states is attributed to the coupling between the $\mathrm{Cu}-\mathrm{O}$ planes within the unit cell. The absence of band splitting in cuprates having only one $\mathrm{Cu}-\mathrm{O}$ layer per unit cell gives credence to the hypothesis that there must be some sort of out-of-plane effect on account of interlayer coupling in cuprates where there are more than one $\mathrm{Cu}-\mathrm{O}$ planes per unit cell.

Several authors have emphasized [7-10] the role of intra cell coupling on the electronic spectra of the bilayer cuprates within the framework of a bilayer tight binding Hubbard Hamiltonian. Based on bilayer electronic band structure calculations Chakravarty and collaborators [11] have estimated a highly anisotropic intra cell coupling having the planar momentum dependence of the form

$$
\varepsilon_{k \perp}=-\frac{t_{\perp}}{4}\left(\cos k_{x} a-\cos k_{y} a\right)^{2},
$$

which gives maximum bilayer splitting at $(\pi, 0)$ point of the Brillouin zone.

The influence of third dimension on the photoemission spectra of two dimensional materials has also been pointed out recently. Bansil et al. [12] have analyzed the 
intercell coupling on the basis of simulated ARPES lineshapes in Bi-2212 and concluded that the inter cell hopping induces a broadening to the photoemission spectra which have a characteristic dependence on the momentum $\vec{k}$. Markiewicz et al. [13] have also analyzed the effect of intercell coupling and the resulting $k_{z}$ dispersion in the cuprates within the framework of the one-band tight binding (TB) model Hamiltonian. These authors have obtained various values of tight binding parameters by fitting to the first principles local density approximation (LDA) based band structures, and also with experimental Fermi surfaces obtained by ARPES experiments in optimally doped and overdoped single layer cuprates, $\mathrm{La}_{2-\mathrm{x}} \mathrm{Sr}_{\mathrm{x}} \mathrm{CuO}_{4}$ and $\mathrm{Nd}_{2-\mathrm{x}} \mathrm{Ce}_{\mathrm{x}} \mathrm{CuO}_{4}$ and bilayer cuprate $\mathrm{Bi}-2212$.

The out-of-plane coupling not only connects the $\mathrm{Cu}-\mathrm{O}$ planes within the unit cell (intra unit cell) but also $\mathrm{Cu}-\mathrm{O}$ planes of the adjacent unit cells (inter unit cell). In bilayer cuprates due to small separation (of the order of 3.2 $\AA$ ) between two $\mathrm{Cu}-\mathrm{O}$ planes within the unit cell, the intra unit cell coupling between two $\mathrm{Cu}-\mathrm{O}$ bilayers results in the band splitting as observed in ARPES measurements. On the other hand, the inter unit cell coupling that connects the two adjacent $\mathrm{Cu}-\mathrm{O}$ planes in different unit cells is believed to be weaker due to greater separation (of the order of $12 \AA$ in Bi-2212 cuprates) between $\mathrm{Cu}-\mathrm{O}$ planes of two adjacent unit cells. Because of very low strength of inter unit cell interaction between $\mathrm{Cu}-\mathrm{O}$ planes, their effect shows up only in recent high resolution ARPES measurements of Chuang et al. [5,14] on Bi-2212 system.

Several mechanisms have been proposed so far to explain the out of plane (c-axis) electronic conduction in multilayer cuprates $[15,16]$ and we are still far from a clear understanding of c-axis electronic conduction in cuprates.

Recently, the influence of inter unit cell resonant tunneling between the copper-oxygen planes on c-axis electronic conductivity in normal state of optimally doped bilayer cuprates is investigated [17]. It is concluded that the c-axis conductivity increases with the increment in intercell resonant tunneling and this effect becomes more prominence at low temperatures $\left(T<300^{\circ} \mathrm{K}\right)$. Therefore, the resonant tunneling mechanism is able to account for out of plan (c-axis) electronic conduction processes in systems like Y-123 and $\mathrm{Bi}-2212$. Most of the recent theoretical studies related to ARPES electronic spectra are based on the two dimensional tight binding Hubbard model and its extension within numerical and analytical approaches and the role of third dimensional coupling on the spectra in the presence of electrons correlation effect has not been clearly understood so far. Therefore, it would be interesting to study the effect of intra cell coupling, inter unit cell resonant tunneling, and electronic correlations simultaneously on the spectral function in doped multilayer cuprates in normal state.

\section{Theoretical Formulation}

For the system like Bi-2212 and Y-123 which have two $\mathrm{Cu}-\mathrm{O}$ planes per unit cell, we have proposed a model Hamiltonian that incorporates the intra cell coupling, inter cell resonant tunneling alongwith strong Coulomb interaction that exist in cuprates.

The mechanism of inter unit cell resonant tunneling is schematically depicted in Figure 1 for Bi-2212 and Y-123 systems. The model Hamiltonian for cuprates with two $\mathrm{Cu}-\mathrm{O}$ planes per unit cell which incorporates inter cell resonant tunneling is given below: (Equations 1(a) and (b)) where, $m$ is unit cell indice and $r, s$ are layer indices within $a$ unit cell (where $r=1, s=2$ and vice-versa). $c_{m r k \sigma}^{+}\left(c_{m r k \sigma}\right)$ are creation (annihilation) operator for the holes of $m^{\text {th }}$ unit cell, $r^{\text {th }} \mathrm{Cu}-\mathrm{O}$ plane with wave vector $\mathrm{k}$ and spin $\sigma$.The $1^{\text {st }}$ term in 1(a) is kinetic energy of charge carrier (holes) within the $\mathrm{Cu}-\mathrm{O}$ plane. The in plane band dispersion $\varepsilon_{r k}$ having the contribution of nearest neighbour hopping $(t)$ and next nearest neighbour hopping $\left(t^{\prime}\right)$ is taken as

$$
\left\{-2 t\left(\cos k_{x} a+\cos k_{y} a\right)-4 t^{\prime}\left(\cos k_{x} a \cos k_{y} a\right)\right\}
$$

It also includes the chemical potential of coupled bilayer system and therefore, initially we have taken Fermi energy $E_{F}=0$ for our calculations. The $2^{\text {nd }}$ term in 1 (a) is the on site Coulomb energy at $\mathrm{Cu} 3 \mathrm{~d}^{9}$ site and in cuprates this energy is quiet large (in the range 5 to $10 \mathrm{eV}$ ) as compared to hopping energy. The $3^{\text {rd }}$ term in $1(a)$ is the single particle coupling between the bilayers within the unit cell and we have assumed momentum dependent of the form:

$$
\begin{aligned}
& H=H_{\text {intracell }}+H_{\text {intercell }}, \text { Here, } H_{\text {intracell }}= \\
& \sum_{m=1,2}\left[\sum_{k, \sigma} \varepsilon_{r k} c_{m r k \sigma}^{+} c_{m r k \sigma}+U \sum_{k, k^{\prime}, q, r} c_{m r k+q \uparrow}^{+} c_{m r k^{\prime} \uparrow} c_{m r k^{\prime}-q \downarrow}^{+} c_{m r k \downarrow}+\sum_{r \neq s, k, \sigma} \varepsilon_{k \perp}\left(c_{m r k \sigma}^{+} c_{m s k \sigma}+c_{m s k \sigma}^{+} c_{m r k \sigma}\right)\right] \\
& H_{\text {intercell }=} \varepsilon_{0} \sum_{l} b_{l}^{+} b_{l}+\sum_{m, r, s, k, \sigma, l} T_{r s}(m, m+1)\left[\left(c_{m r k \sigma}^{+} b_{l}+b_{l}^{+} c_{m+1 s k \sigma}\right)+\left(c_{m+1 s k \sigma}^{+} b_{l}+b_{l}^{+} c_{m r k \sigma}\right)\right]
\end{aligned}
$$




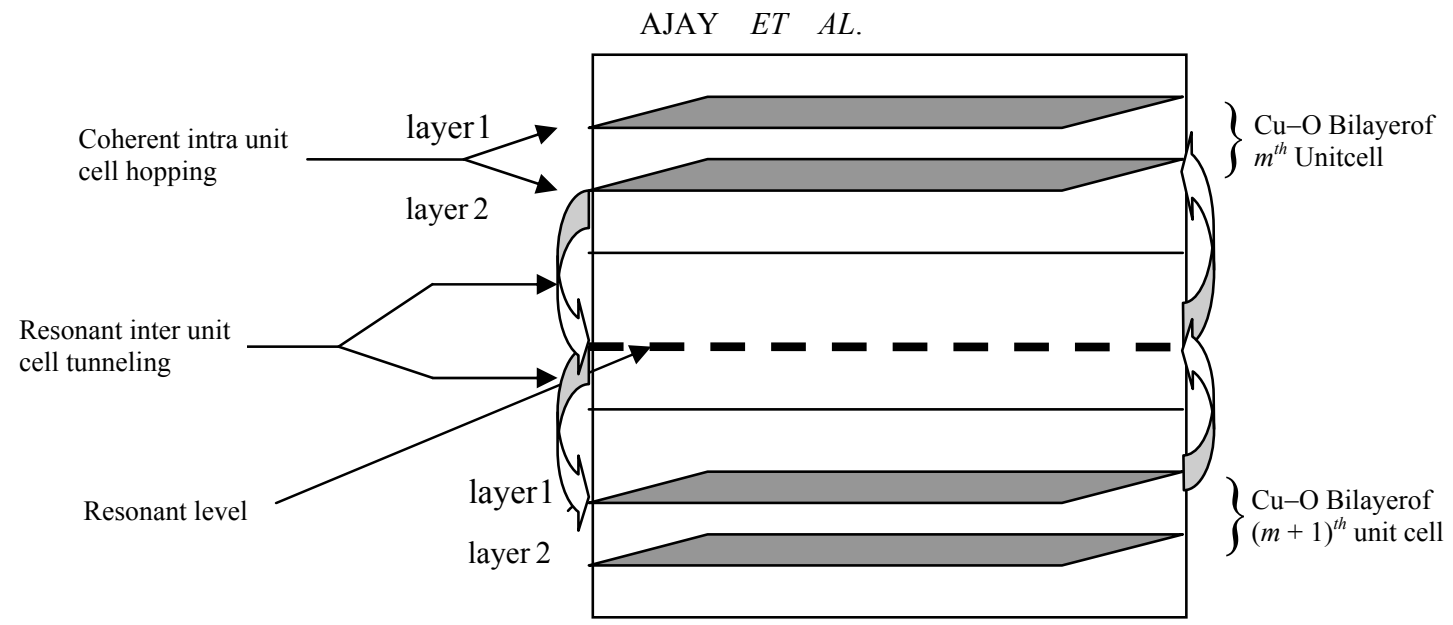

Figure 1. Schematic presentation of intra cell coupling and inter unit cell resonant tunneling in bilayer cuprates (Bi-2212 and Y-123).

$$
\varepsilon_{k \perp}=-\frac{t_{\perp}}{4}\left(\cos k_{x} a-\cos k_{y} a\right)^{2}
$$

as suggested by band structure calculation [11] and ARPES measurements $[3,4,12,13]$.

The $1^{\text {st }}$ term in 1 (b) represents the energy of the resonant level due to oxygen deficient $\mathrm{BiO}$ chain which lies mid way between the two $\mathrm{Cu}-\mathrm{O}$ planes lying in adjoining unit cells. The $2^{\text {nd }}$ term in 1 (b) represents the single particle resonant tunneling from lower layer (say $r^{\text {th }}$ layer) of $m^{\text {th }}$ unit cell to upper layer (say $s^{\text {th }}$ layer) of adjacent $(m+1)^{t h}$ unit cell and vice-versa. The parameter $T_{r s}(m$, $m+1)$ represent the inter unit cell resonant tunneling matrix element for the case when tunneling probability between two $\mathrm{Cu}-\mathrm{O}$ planes in different unit cells is of the order of unity [18]. During the theoretical calculation $T_{r s}(m, m+1)$ is taken as $T_{12}$ for the case of nearest $\mathrm{CuO}$ planes in adjacent unit cells (Figure 1). To obtain an expression for the corresponding Green's function $G_{11}(k, \omega)=\left\langle\left\langle c_{11 k \sigma} \mid c_{11 k \sigma}^{+}\right\rangle\right\rangle$and hence planar single particle spectral function $A(k, \omega)$ for the model Hamiltonian (1), we have employed Green's function equations of motion approach $[9,15,16]$. Finally, we derived a set of coupled equations having higher order Green's functions as:

$$
\begin{gathered}
\left(\omega-\varepsilon_{k}\right)\left\langle\left\langle c_{11 k \sigma} \mid c_{11 k \sigma}^{+}\right\rangle\right\rangle=\frac{1}{2 \pi}+\sum_{k_{1}^{\prime} q} U\left\langle\left\langle c_{11 k_{1}^{\prime} \sigma} c_{11 k_{1}^{\prime}-q-\sigma}^{+} c_{11 k-q-\sigma} \mid c_{11 k \sigma}^{+}\right\rangle\right\rangle \\
+\varepsilon_{k \perp}\left\langle\left\langle c_{12 k \sigma} \mid c_{11 k \sigma}^{+}\right\rangle\right\rangle+T_{12}\left\langle\left\langle b_{1} \mid c_{11 k \sigma}^{+}\right\rangle\right\rangle \\
\left(\omega-\varepsilon_{k}\right)\left\langle\left\langle c_{12 k \sigma} \mid c_{11 k \sigma}^{+}\right\rangle\right\rangle=\varepsilon_{k \perp}\left\langle\left\langle c_{11 k \sigma} \mid c_{11 k \sigma}^{+}\right\rangle\right\rangle+\sum_{k_{1}^{\prime} q} U\left\langle\left\langle c_{12 k_{1}^{\prime} \sigma} c_{12 k^{\prime}-q_{1} \sigma}^{+} c_{12 k-q-\sigma} \mid c_{11 k \sigma}^{+}\right\rangle\right\rangle \\
\left(\omega-\varepsilon_{0}\right)\left\langle\left\langle b_{1} \mid c_{11 k \sigma}^{+}\right\rangle\right\rangle=T_{12}\left(\left\langle\left\langle c_{22 k \sigma} \mid c_{11 k \sigma}^{+}\right\rangle\right\rangle+\left\langle\left\langle c_{11 k \sigma} \mid c_{11 k \sigma}^{+}\right\rangle\right\rangle\right) \\
\left(\omega-\varepsilon_{\mathrm{k}}\right)\left\langle\left\langle\mathrm{c}_{22 \mathrm{k} \sigma} \mid \mathrm{c}_{11 \mathrm{k} \sigma}^{+}\right\rangle\right\rangle=\varepsilon_{\mathrm{k} \perp}\left\langle\left\langle\mathrm{c}_{21 \mathrm{k} \sigma} \mid \mathrm{c}_{11 \mathrm{k} \sigma}^{+}\right\rangle\right\rangle+\sum_{\mathrm{k}_{1}^{\prime} \mathrm{q}} \mathrm{U}\left\langle\left\langle\mathrm{c}_{22 \mathrm{k}_{1}^{\prime} \sigma} \mathrm{c}_{22 \mathrm{k}_{1}^{\prime}-\mathrm{q}-\sigma}^{+} \mathrm{c}_{22 \mathrm{k}-\mathrm{q}-\sigma} \mid \mathrm{c}_{11 \mathrm{k} \sigma}^{+}\right\rangle\right\rangle+\mathrm{T}_{12}\left\langle\left\langle\mathrm{~b}_{1} \mid \mathrm{c}_{11 \mathrm{k} \sigma}^{+}\right\rangle\right\rangle
\end{gathered}
$$

and

$$
\left(\omega-\varepsilon_{k}\right)\left\langle\left\langle c_{21 k \sigma} \mid c_{11 k \sigma}^{+}\right\rangle\right\rangle=\varepsilon_{k \perp}\left\langle\left\langle c_{22 k \sigma} \mid c_{11 k \sigma}^{+}\right\rangle\right\rangle+\sum_{k_{1}^{\prime} q} U\left\langle\left\langle c_{21 k_{1}^{\prime} \sigma} c_{21 k_{1}^{\prime}-q-\sigma}^{+} c_{21 k-q-\sigma} \mid c_{11 k \sigma}^{+}\right\rangle\right\rangle
$$

In order to take into account the electronic correlation effects that exist in cuprates, the equations of motion of higher order Green's functions appearing in Equations
(2-6) can be rewritten and the decoupling in higher order Green's functions in these equations of motion is made at second stage. During the decoupling of higher order 
Green's function, it is also assumed that the system is paramagnetic. This makes the equations tractable and avoids complications. With these approximations and simplification the higher order Green's functions that exist in equations can now be written as:

$$
\begin{gathered}
\left(\omega-\varepsilon_{k_{1}^{\prime}}\right)\left\langle\left\langle c_{11 k_{1}^{\prime} \sigma} c_{11 k_{1}^{\prime}-q-\sigma}^{+} c_{11 k-q-\sigma} \mid c_{11 k \sigma}^{+}\right\rangle\right\rangle=\frac{\delta_{k_{1}^{\prime} k}}{2 \pi}\left\langle c_{11 k_{1}^{\prime}-q-\sigma}^{+} c_{11 k-q-\sigma}\right\rangle+\varepsilon_{k_{1}^{\prime} \perp}\left\langle\left\langle c_{11 k_{1}^{\prime}-q-\sigma}^{+} c_{11 k-q-\sigma} c_{12 k_{1}^{\prime} \sigma} \mid c_{11 k \sigma}^{+}\right\rangle\right\rangle \\
+T_{12}\left\langle\left\langle c_{11 k_{1}^{\prime} q \sigma}^{+} c_{11 k q \sigma} b_{1} \mid c_{11 k \sigma}^{+}\right\rangle\right\rangle \\
-\sum_{k_{1}^{\prime} q} U\left\langle\left\langle c_{11 k_{1}^{\prime}-q-\sigma}^{+} c_{11 k_{1}^{\prime}-q-\sigma} c_{11 k_{1}^{\prime} \sigma} c_{11 k_{1}^{\prime}-q-\sigma}^{+} c_{11 k-q-\sigma} \mid c_{11 k \sigma}^{+}\right\rangle\right\rangle
\end{gathered}
$$

The other higher order equations are also obtained and after truncating the hierarchy of still higher order Green's function in the Hartree Fock approximation, we finally obtained set of linear coupled equations of Green's functions. It is assumed that the charge carriers in $\mathrm{Cu}-\mathrm{O}$ planes are equally distributed and also used a paramagnetic situation (i.e. carriers with up spin are equal to carriers with down spin). Solving the above coupled equations, one derives the Green's function $G_{11}(k, \omega)$ as follows;

$$
\begin{aligned}
& G_{11}(k, \omega)=\frac{1}{2 \pi}\left(\omega-\varepsilon_{k}\right)\left(1+F_{k \omega}\right) \times \\
& {\left[\frac{\left(\omega-\varepsilon_{k}\right)^{2}\left(\omega-\varepsilon_{0}\right)-\varepsilon_{k \perp}^{2}\left(\omega-\varepsilon_{0}\right)\left(1+F_{k \omega}\right)^{2}-T_{12}^{2}\left(\omega-\varepsilon_{k}\right)\left(1+F_{k \omega}\right)}{\left\{\left(\omega-\varepsilon_{k}\right)^{2}-\varepsilon_{k \perp}^{2}\left(1+F_{k \omega}\right)^{2}\right\}\left\{\left(\omega-\varepsilon_{k}\right)^{2}\left(\omega-\varepsilon_{0}\right)-\varepsilon_{k \perp}^{2}\left(\omega-\varepsilon_{0}\right)\left(1+F_{k \omega}\right)^{2}-2 T_{12}^{2}\left(\omega-\varepsilon_{k}\right)\left(1+F_{k \omega}\right)\right\}}\right]}
\end{aligned}
$$

In the absence of Coulomb energy term (i.e. $U=0)$, the

Equation (8), can be written in the following form.

$$
\begin{aligned}
& G_{11}(k, \omega)=\left\langle\left\langle c_{11 k \sigma} \mid c_{11 k \sigma}^{+}\right\rangle\right\rangle \\
& =\frac{1}{2 \pi}\left(\omega-\varepsilon_{k}\right) \times\left[\frac{\left(\omega-\varepsilon_{0}\right)\left\{\left(\omega-\varepsilon_{k}\right)^{2}-\varepsilon_{k \perp}^{2}\right\}-T_{12}^{2}\left(\omega-\varepsilon_{k}\right)}{\left\{\left(\omega-\varepsilon_{k}\right)^{2}-\varepsilon_{k \perp}^{2}\right\}\left[\left(\omega-\varepsilon_{0}\right)\left\{\left(\omega-\varepsilon_{k}\right)^{2}-\varepsilon_{k \perp}^{2}\right\}-2 T_{12}^{2}\left(\omega-\varepsilon_{k}\right)\right]}\right]
\end{aligned}
$$

If, in the above expression of $G_{11}(k, \omega)$, the resonant tunneling is taken as zero $\left(T_{12}=0\right)$, we obtain the propagator for bilayer system having intra cell coupling only, and Equation (8) reduced to the following:

$$
\begin{aligned}
G_{11}(k, \omega)= & \frac{1}{2 \pi}\left[\frac{\omega-\varepsilon_{k}}{\left(\omega-\varepsilon_{k}\right)^{2}-\varepsilon_{k \perp}^{2}}\right] \quad \text { can rewrite the Equation (8) as: } \\
& G_{11}(k, \omega)=\frac{1}{2 \pi}\left(\omega-\varepsilon_{k}\right)\left(1+F_{k \omega}\right)\left\{\frac{\omega^{3}+A \omega^{2}+B \omega+C}{\omega^{5}+D \omega^{4}+E \omega^{3}+F \omega^{2}+G \omega+H}\right\}
\end{aligned}
$$

One can check from above Equation (10) that there are two quasiparticle energy branches (i.e. $\omega_{1,2}=\varepsilon_{k} \pm \varepsilon_{k \perp}$ ): a manifestation of intrabilayer coupling between two $\mathrm{CuO}_{2}$ planes within the unit cell. On simplification one

where,

$$
\begin{aligned}
& A=-\left(\varepsilon_{0}+2 \varepsilon_{k}\right) \\
& B=2 \varepsilon_{k} \varepsilon_{0}+\varepsilon_{k}^{2}-\varepsilon_{k \perp}^{2}\left(1+F_{k \omega}\right)^{2}-T_{12}^{2}\left(1+F_{k \omega}\right) \\
& C=-\left\{\varepsilon_{k}^{2} \varepsilon_{0}-\varepsilon_{k \perp}^{2} \varepsilon_{0}\left(1+F_{k \omega}\right)^{2}-\varepsilon_{k} T_{12}^{2}\left(1+F_{k \omega}\right)\right\} \\
& D=-\left(\varepsilon_{0}+4 \varepsilon_{k}\right)
\end{aligned}
$$




$$
\begin{aligned}
& E=4 \varepsilon_{k} \varepsilon_{0}+6 \varepsilon_{k}^{2}-2 \varepsilon_{k \perp}^{2}\left(1+F_{k \omega}\right)^{2}-2 T_{12}^{2}\left(1+F_{k \omega}\right) \\
& F=-\left\{6 \varepsilon_{k}^{2} \varepsilon_{0}+4 \varepsilon_{k}^{3}-4 \varepsilon_{k} \varepsilon_{k \perp}{ }^{2}\left(1+F_{k \omega}\right)^{2}-2 \varepsilon_{0} \varepsilon_{k \perp}{ }^{2}\left(1+F_{k \omega}\right)^{2}-6 \varepsilon_{k} T_{12}{ }^{2}\left(1+F_{k \omega}\right)\right\} \\
& G=\left\{\begin{array}{l}
4 \varepsilon_{k}^{3} \varepsilon_{0}-2 \varepsilon_{k}^{2} \varepsilon_{k \perp}^{2}\left(1+F_{k \omega}\right)^{2}+\varepsilon_{k}^{4}-4 \varepsilon_{k} \varepsilon_{0} \varepsilon_{k \perp}{ }^{2}\left(1+F_{k \omega}\right)^{2}-6 \varepsilon_{k}^{2} T_{12}^{2}\left(1+F_{k \omega}\right)+ \\
\varepsilon_{k \perp}{ }^{4}\left(1+F_{k \omega}\right)^{4}+2 T_{12}^{2} \varepsilon_{k \perp}^{2}\left(1+F_{k \omega}\right)^{3}
\end{array}\right\}
\end{aligned}
$$

and

$$
H=-\left\{\begin{array}{l}
\varepsilon_{k}{ }^{4} \varepsilon_{0}-2 \varepsilon_{k}{ }^{2} \varepsilon_{0} \varepsilon_{k \perp}{ }^{2}\left(1+F_{k \omega}\right)^{2}-2 \varepsilon_{k}{ }^{3} T_{k 12}{ }^{2}\left(1+F_{k \omega}\right)+\varepsilon_{k \perp}{ }^{4} \varepsilon_{0}\left(1+F_{k \omega}\right)^{4} \\
+2 \varepsilon_{k \perp}{ }^{2}\left(1+F_{k \omega}\right)^{3} \varepsilon_{k} T_{12}{ }^{2}
\end{array}\right\}
$$

Using simple algebra above Equation (12) can be rearranged as:

$$
G_{11}(k, \omega)=\frac{1}{2 \pi} \sum_{i=1}^{5} \frac{A_{i}}{\left(\omega-\alpha_{i}\right)}
$$

where, $\alpha_{1} \ldots \ldots \ldots \alpha_{5}$ are the five quasi-particle energies which have been computed numerically. The planar single particle spectral function $A(k, \omega)$ can be calculated from the above Green's function $G_{11}(k, \omega)$ numerically by using the relationship:

$$
A(k, \omega)=-\frac{1}{\pi} \operatorname{Im} G_{11}(k, \omega),
$$

where Im stands for imaginary part of Green's function. Using Equations (12) and (13) the expression for planar electronic spectral function can be written in the following form:

$$
A(k, \omega)=\frac{1}{\pi} \sum_{i=1}^{5} \frac{\Gamma A_{i}}{\Gamma^{2}+\left(\omega-\alpha_{i}\right)^{2}}
$$

To fit the line shape of the photoemission experimental results, we need to solve $\delta$-functions involved in the spectral function (Equation (14)). The calculation of $A(k, \omega)$ would give a broadening of the quasiparticle peak. A Lorentizian type of broadening is used as given below:

$$
\delta\left(\omega-\tilde{\varepsilon}_{k}\right) \cong \operatorname{Lim}_{\Gamma \rightarrow 0} \frac{1}{\pi} \frac{\Gamma}{\left(\omega-\tilde{\varepsilon}_{k}\right)^{2}+\Gamma^{2}}
$$

The phenomenological broadening parameter $\Gamma$ takes care of quasi-particle scattering rate and initially taken to be independent of $k$ and $\omega[19,20]$ as we are interested in the influence of inter unit cell resonant tunneling on photoemission spectral function. The line shape of the peak in the ARPES spectral function $A(k, \omega)$ can be analyzed using Equation (14) numerically.

\section{Results and Discussion}

The spectral function $A(k, \omega)$ is measured by angle resolved photoemission spectroscopy. The measured
$A(k, \omega)$ can be numerically analyzed using Equations (13-15). The $A(k, \omega)$ is analyzed especially at $(\pi, 0)$ points of the Brillouin zone, where the splitting in the ARPES spectra of Bi2212 is found to be maximum in normal state. These calculations would be relevant to bilayer cuprates, $\mathrm{Bi}-2212$, because the range of various intra and inter cell parameters used in the present analysis have been taken from recent electronic band structure calculations $[10,11]$ and works on electronic spectra of Bi2212 cuprates $[3,4,12,13]$.

In Figure 2, one can observe the spectral function $A(k, \omega)$ against energy $(\omega)$ for different intra cell coupling strength in optimal doped regime of $\mathrm{Bi}-2212$ in the normal state in the presence of inter cell resonant tunneling $\left(T_{12}=0.1 \mathrm{eV}\right)$ and for the case $\left.U=0.0 \mathrm{eV}\right)$. The variation of $A(k, \omega)$ versus band energy $(\omega)$ at $(\pi, 0)$ point of Brillouin zone shows a splitting in the spectral function in the presence of intra cell coupling between the planes $\left(t_{\perp}=0.2 \mathrm{eV}\right)$. Such a bilayer splitting in $\mathrm{Bi}-2212$ at $(\pi, 0)$ point is also observed in recent ARPES measurements [4-7]. On introducing the inter unit cell resonant tunneling $\left(T_{12}=0.05 \mathrm{eV}, 0.075 \mathrm{eV}\right.$ and $0.1 \mathrm{eV}$ and keeping $t_{\perp}=0.1 \mathrm{eV}$ ), one can point out a broadening in the spectral function (Figure 3). Also, the bilayer splitting in the spectral function disappeared due to broadening in the spectra on increasing inter cell resonant tunneling term $\left(T_{12}=0.1 \mathrm{eV}\right.$ to $0.15 \mathrm{eV}$ ) (Figure 4). Such broadening in the normal state spectra has also been predicted by simulated ARPES line shapes in Bi-2212 bilayer systems as manifestation of third dimensional inter unit cell coupling $[12,13]$. On comparing Fig. 2 and Figure 4 one can observe that intra cell coupling $\left(t_{\perp}\right)$ leads the bilayer splitting in the spectral function, while inter unit cell resonant tunneling $\left(T_{12}\right)$ suppresses the bilayer splitting and induces broadening in the spectral peak close to Fermi level.

One can also analyze the influence of inter cell resonant tunneling term $\left(T_{12}\right)$ on the spectral function at $(\pi, 0)$ point in the presence of Coulomb correlation $(U)$, keeping other parameters fixed, and using Green's func- 


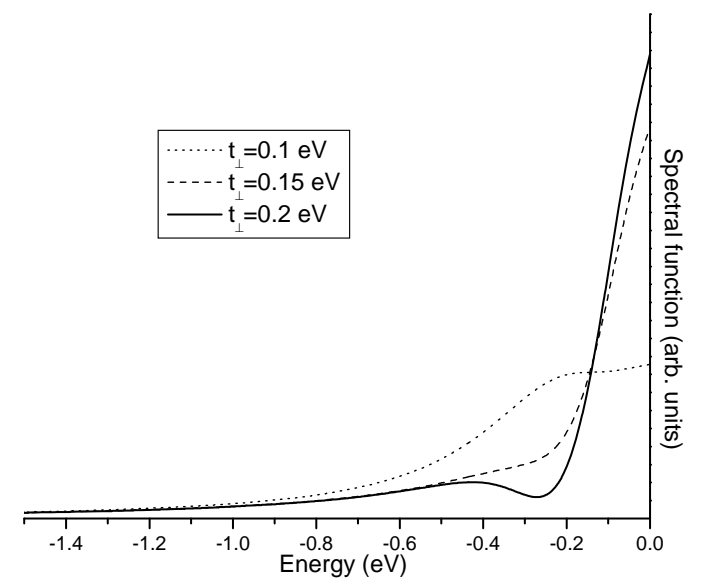

Figure 2. Spectral function versus energy at $(\pi, 0)$ point of the Brillouin zone keeping $t=0.4 \mathrm{eV}, T_{12}=0.1 \mathrm{eV}, U=0.0 \mathrm{eV}$, $n=0.2, \Gamma=0.25 \mathrm{eV}$, and $\varepsilon_{0}=0.05 \mathrm{eV}$.

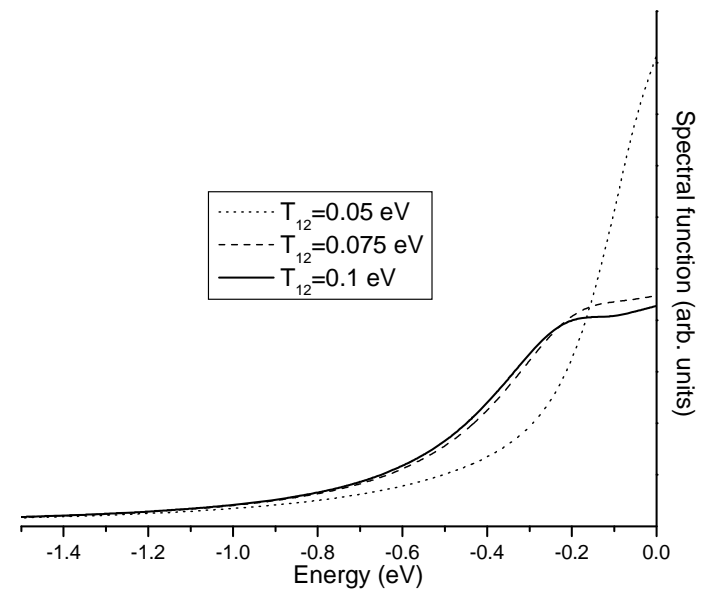

Figure 3. Spectral function versus energy at $(\pi, 0)$ point of the Brillouin zone keeping $t=0.4 \mathrm{eV}, t_{\perp}=0.1 \mathrm{eV}, U=0.0$ $\mathrm{eV}, \boldsymbol{n}=\mathbf{0 . 2}, \Gamma=0.25 \mathrm{eV}$, and $\varepsilon_{0}=0.05 \mathrm{eV}$.

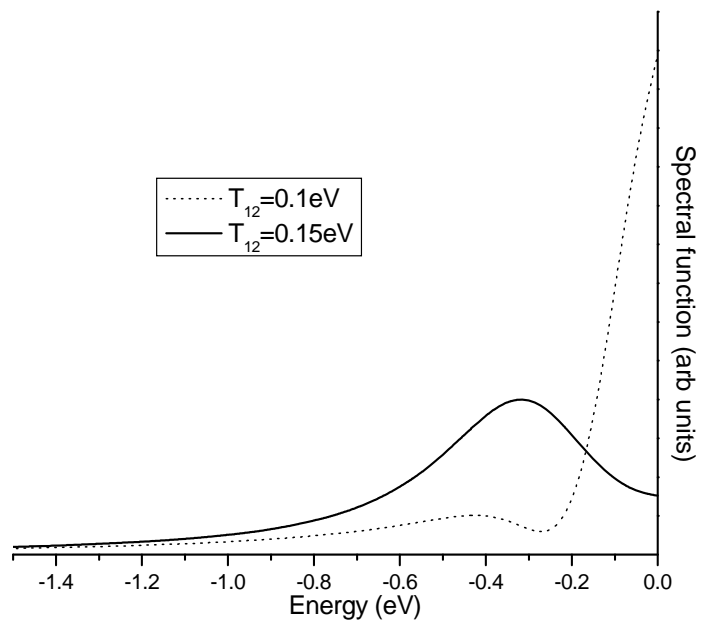

Figure 4. Spectral function versus energy at $(\pi, 0)$ point of the Brillouin zone keeping $t=0.4 \mathrm{eV}, t_{\perp}=0.2 \mathrm{eV}, U=0.0$ $\mathrm{eV}, \boldsymbol{n}=0.2, \Gamma=0.25 \mathrm{eV}$, and $\varepsilon_{0}=0.05 \mathrm{eV}$.

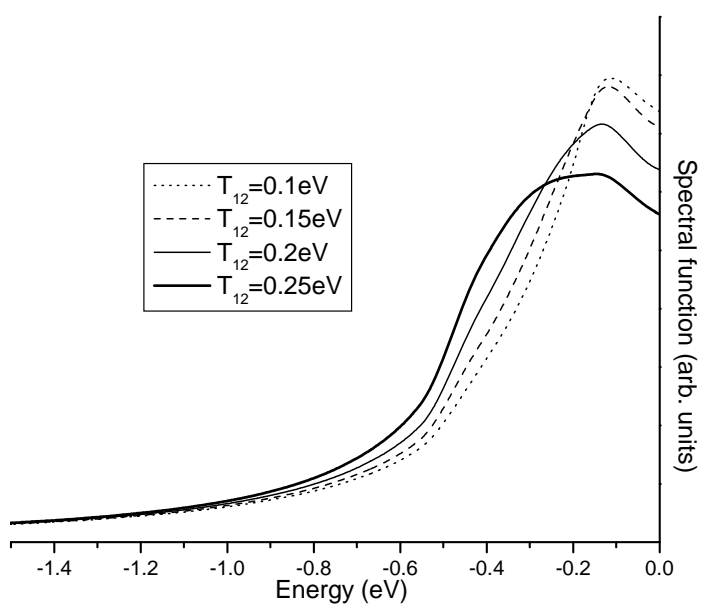

Figure 5. Spectral function versus energy at $(\pi, 0)$ point of the Brillouin zone keeping $\boldsymbol{t}=\mathbf{0 . 4} \mathrm{eV}, t_{\perp}=0.2 \mathrm{eV}, \boldsymbol{U}=3.0$ $\mathrm{eV}, \boldsymbol{n}=\mathbf{0 . 2}, \Gamma=0.25 \mathrm{eV}$, and $\varepsilon_{0}=0.05 \mathrm{eV}$.

tion Equation (18). In Figure 5, the spectral function versus energy for different values of inter cell resonant tunneling term $\left(T_{12}=0.1 \mathrm{eV}\right.$ to $\left.0.25 \mathrm{eV}\right)$ in the presence of Coulomb correlation (i.e. $U=3 \mathrm{eV}$ ) have been plotted. It is pointed out from the Figure 5 that in the presence of both the electronic correlations, and inter cell resonant tunneling $\left(T_{12}\right)$ the broadening in spectra [1-4] is more as compare to the absence of Coulomb correlations (Figures 3 and 4).

Finally, it is concluded that the intra unit cell coupling is responsible for bilayer splitting of electronic spectra, while the inter unit cell resonant tunneling leads to broadening in the spectral features especially at $(\pi, 0)$ point of optimally doped bilayer (Bi-2212) cuprates and in good correspondence with recent ARPES measurements [12]. The electron correlations also play an important role in determining the shape of spectral function. As the antinodal region of hole doped cuprates is so called hot spot, in which the electron-electron correlation broaden the spectral function. Besides, there may be another source to broaden the spectral function like as randomness in the $\mathrm{CuO}_{2}$ planes alongwith inter cell incoherent resonant tunneling and carrier concentration. Therefore, the presence of finite electron correlations, the inter unit cell resonant tunneling $\left(T_{12}\right)$ suppresses the bilayer splitting in the spectral function due to broadening in the spectral features close to Fermi level in optimal doped bilayer cuprates.

\section{Acknowledgements}

We are highly thankful to Prof. S. K. Joshi, National Physical Laboratory, New Delhi, India for his valuable suggestions and encouragement. 


\section{References}

[1] M. R. Norman and C. Pepin, "The Electronic Nature of High Temperature Cuprate Superconductors," Reports on Progress in Physics, Vol. 66, No. 10, September 2003, p. 1547. doi:10.1088/0034-4885/66/10/R01

[2] A. Damascelli, Z. Hussain and Z. X. Shen, "Angle-Resolved Photoemission Studies of the Cuprate Superconductors," Reviews of Modern Physics, Vol. 75, No. 2, April 2003, pp. 473-541. doi:10.1103/RevModPhys.75.473

[3] D. L. Feng, N. P. Armitage, D. H. Lu, A. Damascelli, J. P. Hu, P. Bogdanov, A. Lanzara, F. Ronning, K. M. Shen, H. Eisaki, C. Kim, J. I. Shinoyama, H. Kishio and Z.-X. Shen, "Bilayer Splitting in the Electronic Structure of Heavily Overdoped $\mathrm{Bi}_{2} \mathrm{Sr}_{2} \mathrm{CaCu}_{2} \mathrm{O}_{8+\delta}$," Physical Review Letters, Vol. 86, No. 24, June 2001, pp. 5550-5553. doi:10.1103/PhysRevLett.86.5550

[4] D. L. Feng, A. Damascelli, K. M. Shen, N. Motoyama, D. H. Lu, H. Eisaki, K. Shimizu, J.-I. Shimoyama, K. Kishio, N. Kaneko, F. Ronning, N. P. Armitage and Z.-X .Shen, "Electronic Structure of the Trilayer Cuprate Superconductor $\mathrm{Bi}_{2} \mathrm{Sr}_{2} \mathrm{Ca}_{2} \mathrm{Cu}_{3} \mathrm{O}_{10+\delta}$," Physical Review Letters, Vol. 88, No. 10, February 2002, Article ID: 107001.

[5] Y. D. Chaung, A. D. Gromko, A. Fedorov, Y. Aiura, K. Oka, Y. Ando, H. Eisaki, S. I. Uchida and D. S. Dessau, "Doubling of the Bands in Overdoped $\mathrm{Bi}_{2} \mathrm{Sr}_{2} \mathrm{CaCu}_{2} \mathrm{O}_{8+\delta}$. Evidence for $c$-Axis Bilayer Coupling," Physical Review Letters, Vol. 87, No. 11, August 2001, Article ID: 117002.

[6] A. A. Kordyuk, S. V. Borisenko, T. K. Kim, K. Nenkov, M. Knupfer, M. S. Golden, J. Fink, H. Berger, R. Follath, "Origin of the Peak-Dip-Hump Line Shape in the Superconducting - State $(\pi, 0)$ Photoemission Spectra of $\mathrm{Bi}_{2} \mathrm{Sr}_{2} \mathrm{CaCu}_{2} \mathrm{O}_{8}$," Physical Review Letters, Vol. 89, No. 7, July 2002, Article ID: 077003, pp. 1-4.

[7] A. I. Liechtenstein, O. Gunnarson, O. K. Anderson and R. M. Martin, "Quasiparticle Bands and Superconductivity in Bilayer Cuprates," Physical Review B, Vol. 54, No. 17, November 1996, pp. 12505-12508. doi:10.1103/PhysRevB.54.12505

[8] Y. H. Su, J. Chang, H. T. Lu, H. G. Luo and T. Xiang, "Effect of Bilayer Coupling on Tunneling Conductance of Double-Layer High- $T_{c}$ Cuprates," Physical Review B, Vol. 68, No. 21, December 2003, Article ID: 212501. doi:10.1103/PhysRevB.68.212501

[9] Ajay, "Role of Interlayer Coupling in the Superconducting State of Layered Cuprate Superconductors," Physica C: Superconductivity, Vol. 316, No. 3-4, May 1999, pp. 267-272. doi:10.1016/S0921-4534(99)00219-1

[10] S. Chakravarty, K. Hae-Young and E. Abraham, "Frustrated Kinetic Energy, the Optical Sum Rule, and the Mechanism of Superconductivity," Physical Review Let- ters, Vol. 82, No. 11, March 1999, pp. 2366-2369. doi:10.1103/PhysRevLett.82.2366

[11] S. Chakravarty, A. Sudbo, P. W. Anderson and S. Strong, "Interlayer Tunneling and Gap Anisotropy in HighTemperature Superconductors," Science, Vol. 261, No. 5119, July 1993, pp. 337-340.

doi:10.1126/science.261.5119.337

[12] A. Bansil, M. Lindroos, S. Sahrakorpi and R. S. Markiewicz, "Influence of the Third Dimension of Quasi-Two-Dimensional Cuprate Superconductors on Angle-Resolved Photoemission Spectra," Physical Review B, Vol. 71, No. 1, January 2005, Article ID: 012503.

[13] R. S. Markiewicz, S. Sahrakorpi, M. Lindroos, H. Lin and A. Bansil, "One-Band Tight-Binding Model Parametrization of the High- $T_{c}$ Cuprates Including the Effect of $K_{z}$ Dispersion," Physical Review B, Vol. 72, No. 5, August 2005, Article ID: 054519. doi:10.1103/PhysRevB.72.054519

[14] Y. D. Chaung, A. D.Gromko, A. V. Fedorov, Y. Aiura, K. Oka, Y. Ando, M. Lindroos, R. S. Markieweiz, A. Bansil and D. S. Dessau, "Bilayer Splitting and Coherence Effects in Optimal and Underdoped $\mathrm{Bi}_{2} \mathrm{Sr}_{2} \mathrm{CaCu}_{2} \mathrm{O}_{8+\delta}$," Physical Review B, Vol. 69, No. 9, March 2004, Article ID: 094515, pp. 1-7.

[15] R. Lal, Ajay, R. L. Hota and S. K. Joshi, "Model for $c$-Axis Resistivity of Cuprate Superconductors," Physical Review B, Vol. 57, No. 10, March 1998, pp. 6126-6136. doi:10.1103/PhysRevB.57.6126

[16] Ajay, A. Pratap and S. K. Joshi, "Role of $\mathrm{Cu} \mathrm{d-d} \mathrm{In-}$ ter-Orbital Electron Correlation on the out-of-Plane Conduction in Cuprates," Physica C: Superconductivity, Vol. 371, No. 2, June 2002, pp. 139-145. doi:10.1016/S0921-4534(01)01085-1

[17] B. S. Tewari, A. Dhyani and Ajay, "Influence of Inter Cell Resonant Tunneling on the out-of-Plane Electronic Transport Behavior in Layered High $T_{c}$ Cuprates," European Physical Journal B, Vol. 66, No. 1, October 2008, pp. 67-74. doi:10.1140/epjb/e2008-00375-6

[18] A. A. Abrikosov, "Resonant Tunneling in High- $\mathrm{T}_{\mathrm{c}} \mathrm{Su}-$ perconductors," Physica C: Superconductivity, Vol. 317, May 1999, pp. 154-174. doi:10.1016/S0921-4534(99)00056-8

[19] P. W. Leung, B. O. Wells and R. J. Gooding, "Comparison of 32-Site Exact-Diagonalization Results and ARPES Spectral Functions for the Antiferromagnetic Insulator $\mathrm{Sr}_{2} \mathrm{CuO}_{2} \mathrm{Cl}_{2}$," Physical Review B, Vol. 56, No. 10, 1997, pp. 6320-6326. doi:10.1103/PhysRevB.56.6320

[20] T. Valla, A. V. Fedorov, P. D. Johnson, B. O. Wells, S. L. Hulbert, Q. Li, G. D. Gu and N. Koshizuka, "Evidence for Quantum Critical Behavior in the Optimally Doped Cuprate $\mathrm{Bi}_{2} \mathrm{Sr}_{2} \mathrm{CaCu}_{2} \mathrm{O}_{8+\delta}$," Science, Vol. 285, No. 5436, September 1999, pp. 2110-2113. doi:10.1126/science. 285.5436 .2110 